\title{
A dynamic Parrondo's paradox for continuous seasonal systems
}

\author{
Anna Cima ${ }^{(1)}$, Armengol Gasull ${ }^{(1)}$ and Víctor Mañosa ${ }^{(2)}$ \\ (1) Departament de Matemàtiques, Facultat de Ciències, \\ Universitat Autònoma de Barcelona, \\ 08193 Bellaterra, Barcelona, Spain \\ cima@mat.uab.cat, gasull@mat.uab.cat \\ (2) Departament de Matemàtiques, \\ Universitat Politècnica de Catalunya \\ Colom 11, 08222 Terrassa, Spain \\ victor.manosa@upc.edu
}

\begin{abstract}
We show that planar continuous alternating systems, which can be used to model systems with seasonality, can exhibit a type of Parrondo's dynamic paradox, in which the stability of an equilibrium, common to all seasons is reversed for the global seasonal system. As a byproduct of our approach we also prove that there are locally invertible orientation preserving planar maps that cannot be the time-1 flow map of any smooth planar vector field.
\end{abstract}

Mathematics Subject Classification 2010: Primary: 37C75; 34D20. Secondary: 37C25.

Keywords: Continuous dynamical systems with seasonality, non-hyperbolic critical points, local asymptotic stability, Parrondo's dynamic paradox.

\section{Introduction and Main results}

For dynamical systems given by differential equations, alternating systems take the form

$$
\left\{\begin{array}{l}
\dot{\mathbf{x}}(t)=X_{1}(\mathbf{x}(t)) \text { for } t \text { such that } t(\bmod T) \in\left[0, T_{1}\right), \\
\dot{\mathbf{x}}(t)=X_{2}(\mathbf{x}(t)) \text { for } t \text { such that } t(\bmod T) \in\left[T_{1}, T_{1}+T_{2}\right), \\
\vdots \\
\dot{\mathbf{x}}(t)=X_{n}(\mathbf{x}(t)) \text { for } t \text { such that } t(\bmod T) \in\left[T_{1}+\cdots+T_{n-1}, T_{1}+\cdots+T_{n}\right),
\end{array}\right.
$$


where $T=\sum_{j=1}^{n} T_{j}$, with $T_{j}>0$ for $j=1,2, \ldots, n$, and $X_{j}$ being class $\mathcal{C}^{1}$ vector fields. They can be used to model continuous seasonal systems with $n$ seasons of durations $T_{1}, T_{2}, \ldots, T_{n}$. It is not necessary to recall the importance of these kind of systems in mathematical biology, for instance in population models for which the seasonality has an effect in the reproduction and mortality rates due to environmental circumstances or to human intervention like harvesting, see $[12,19,20]$ and references therein (and $[5,9,14,15]$ for discrete examples); or also in epidemiological models with periodic contact rate, see [3] and the references therein.

The existence of chaotic behaviors in this kind of systems have been reported, see for instance the proof of the existence of topological horseshoes in the Poincaré maps associated to the flow of a 2-seasonal Lotka-Volterra system of type (1) in [18]. The study of chaotic dynamics in continuous seasonal systems is a challenge that will continue to require a lot of attention. In this work we expose a collateral aspect that appears, however, in regular regimes: one type of dynamic Parrondo's paradox.

The so called Parrondo's paradox is a paradox in game theory, that in a few words says that a combination of losing strategies can become a winning strategy, see [11, 16]. Several dynamical versions of related paradoxes are presented in $[4,6,7,8]$ for discrete non-autonomous dynamical systems. In the first paper the authors combine periodically one-dimensional maps $f_{1}$ and $f_{2}$ to give rise to chaos or order. The existence of discrete systems that exhibit (numerically) chaotic dynamics by alternating regular, or more precisely, integrable systems, has been referred in [6] and [7]. In this last reference also are shown alternating systems with regular (integrable) dynamics obtained by alternating an integrable map and a numerically chaotic one. In [8] we study a local problem, but in any dimension. In particular, we relate the stability of a common fixed point of two planar maps, $F_{1}$ and $F_{2}$, with the stability of this point for $F_{2} \circ F_{1}$. We prove that when the fixed points have complex conjugated eigenvalues of modulus 1 (i.e. elliptic fixed points), a common attracting character of the common fixed point of $F_{1}$ and $F_{2}$, can be reversed for $F_{2} \circ F_{1}$. This phenomenon is the one that we named Parrondo's dynamic type paradox for 2-periodic discrete dynamical systems. In this work we will show that a similar dynamical paradox appears for continuous seasonal systems.

Remember that a singular point $\mathbf{p}$ is said to be stable if for every neighborhood $\mathcal{U}$ of $\mathbf{p}$ there is a neighborhood $\mathcal{V} \subset \mathcal{U}$ such that every solution $\varphi\left(t ; \mathbf{p}_{0}\right)$ with $\mathbf{p}_{0} \in \mathcal{V}$ is defined and lies in $\mathcal{U}$ for all $t>0$. If, in addition, $\mathcal{V}$ is such that $\lim _{t \rightarrow+\infty} \varphi\left(t ; \mathbf{p}_{0}\right)=\mathbf{p}$ then the point is (locally) asymptotically stable (LAS from now on). The point is a repeller if it is not stable and $\lim _{t \rightarrow-\infty} \varphi\left(t ; \mathbf{p}_{0}\right)=\mathbf{p},[10]$.

As noted in [3], the asymptotic stability of the equilibria of a seasonal system, for instance the disease-free equilibrium of an epidemiological model, is a more complex issue 
than in the autonomous case. In this note we evidence that a seasonal system of type (1) can exhibit a dynamic-type Parrondo's paradox, in which the stability of an equilibrium common to all stations (either LAS or a repeller), is reversed for the seasonal system (1). That is, we show that there exist systems (1) with a common singular point which is LAS (resp. repeller) for each season system $\dot{\mathbf{x}}=X_{i}(\mathbf{x})$ for $i=1, \ldots, n$ and such it is a repeller (resp. LAS) for the global seasonal system.

To simplify the problem, we prove the existence of the Parrondo's-type paradox for planar differential with two seasons, both with duration $T_{1}=T_{2}=1$. Hence systems of the form

$$
\left\{\begin{array}{l}
\dot{\mathbf{x}}(t)=X_{1}(\mathbf{x}(t)) \text { for } t \in[2 k, 2 k+1), \\
\dot{\mathbf{x}}(t)=X_{2}(\mathbf{x}(t)) \text { for } t \in[2 k+1,2 k+2), k \in \mathbb{N} \cup\{0\},
\end{array}\right.
$$

with $\mathbf{x}(t) \in \mathbb{R}^{2}$. Our main result is:

Theorem 1. There exist planar polynomial vector fields $X_{1}$ and $X_{2}$ sharing a common singular point which is LAS (resp. repeller) for both of their associated differential systems, and such that it is a repeller (resp. LAS) for the 2-seasonal differential system (2).

Notice that this theoretical result opens a practical interesting situation. Let us consider a system where the state variables represent the density of individuals of an age-structured population of a species that can be potentially dangerous to humans, like for instance mosquitoes, [13]. Let us assume that for two different environmental situations (the two seasons) the zero solution is a repeller. Of course, this corresponds to unwanted scenarios since, in each season, for an arbitrary small initial density of individuals the amount of them increases over time. Then, it might happen that alternating both situations we get a system with the origin as a LAS critical point, implying the population decline (and long term extinction) of the dangerous species.

In the following, we will use complex notation in order to simplify the expressions. Hence instead of taking real planar vector fields $U(x, y) \partial / \partial x+V(x, y) \partial / \partial y$ with $(x, y) \in \mathbb{R}^{2}$, we will consider the same vector fields but in complex notation $X(z, \bar{z}) \partial / \partial z+\bar{X}(z, \bar{z}) \partial / \partial \bar{z}$ where $z=x+i y \in \mathbb{C}$, with associated differential equation $\dot{z}=X(z, \bar{z})$, where, of course $\dot{\bar{z}}=\bar{X}(z, \bar{z})$.

One of the key ingredients in our approach will be to know whether for a given local polynomial diffeomorphism of the form

$$
F(z, \bar{z})=\mathrm{e}^{i \alpha} z+\sum_{j+k=2}^{n} f_{j, k} z^{j} \bar{z}^{k}, \quad \alpha \in(0,2 \pi),
$$

of degree at most $n$, that has a non-hyperbolic elliptic fixed point at the origin, there exists 
of a polynomial vector field with

$$
X(z, \bar{z})=i \alpha z+\sum_{j+k=2}^{n} a_{j, k} z^{j} \bar{z}^{k}
$$

and such that its associated flow $\varphi(t ; z, \bar{z})$ satisfies

$$
\varphi(1 ; z, \bar{z})=F(z, \bar{z})+O(n+1)
$$

for every $(z, \bar{z})$, for $z$ in a small enough neighborhood of $z=0$, and where $f(z, \bar{z})=O(k)$ if there exist two constants $\delta>0$ and $C$ such that $|f(z, \bar{z})| \leq C|z|^{k}$ for all $z$ with $|z|<\delta$. As we will see, for our purposes we only will need to consider the cases $n=2$ or $n=3$. This question is solved in next section.

We also would like to comment that very few planar polynomial maps are exactly a flow at a fixed time, i.e. the remainder term $O(n+1)$ in $(5)$ is identically zero. They are the so-called polynomial flows, and the normal forms of their corresponding vector fields are given in [2, Thm. 4.3].

In fact, ultimately, the proof of Theorem 1 relies on the fact that, near a critical point, the flow of some suitable vector fields are such, up to certain fixed order on the initial conditions, their associated time-1 maps are the ones given in Example 7 of [8]. We recall them in Proposition 12. These maps display the features of the Parrondo's dynamic paradox for the dynamics induced by iterating maps and this fact translates to alternating systems of differential equations. This proof is given in Section 3.

As a byproduct of our study we obtain the following result that we believe is interesting by itself. Its proof is given in Section 4 .

Theorem 2. It holds:

(i) Consider a local diffeomorphism of the form (3), where $\mathrm{e}^{i \alpha}$ is not a root of the unity. Then, for any $n \geq 2$ there is a unique polynomial vector field of the form (4) and degree at most $n$ such that its flow satisfies Equation (5).

(ii) For any $n \geq 2$, there exists a map $F$ of the form (3) with $\alpha=2 \pi /(n+1)$ for which there is no $\mathcal{C}^{n+1}$ vector field $X$ whose flow $\varphi(t ; z, \bar{z})$ satisfies Equation (5).

The above result implies the existence of planar local diffeomorphisms, preserving orientation, that can not be given as the flow at a fixed time of smooth planar vector fields. Particular examples of such maps are given in (13). We notice that it is known that the "embedding problem" (that is the answer to the question: can an orientation preserving diffeomorphism be embedded as the time-one map of a flow of an autonomous vector field?) has negative answer in the general setting, see [17] for instance. It is also known that for 
any $k \in \mathbb{N} \cup\{\infty\}$ the subset of local $\mathcal{C}^{k}$ diffeomorphisms having an embedding vector field with some smoothness is dense in the set of all local $\mathcal{C}^{k}$ diffeomorphisms with the coefficient topology, see [21]. Also as a consequence of [22, Theorem 1.3 (a)] all planar $\mathcal{C}^{\infty}$ diffeomorphisms with a hyperbolic fixed point that have a $\mathcal{C}^{\infty}$ embedding flow are characterized. Our result provide a simple proof and a very simple example in the case of non-hyperbolic fixed points, and gives some insight on the open problem about the embedding problem for planar diffeomorphisms with non-hyperbolic fixed points stated in this last reference.

\section{Vector fields with prescribed maps as time-1 map}

\subsection{A recurrent procedure}

We start establishing the structure equation that must satisfy the first terms of a flow map associated with a vector field. First we prove that if a flow map satisfies Equations (3)-(5), then the vector field must have the form $X(z, \bar{z})=i \alpha z+O(2)$, so we will work with vector fields with this fixed linear part.

Lemma 3. A planar $\mathcal{C}^{2}$ vector field $X(z, \bar{z})$ such that its associate flow satisfies $\varphi(1 ; z, \bar{z})=$ $\mathrm{e}^{i \alpha t} z+O(2)$ has the form $X(z, \bar{z})=i \alpha z+O(2)$.

Proof. We set $X(z, \bar{z})=a_{1,0} z+a_{0,1} \bar{z}+O(2)$. By plugging the the associate flow $\varphi(t)=$ $\varphi_{1,0}(t) z+\varphi_{0,1}(t) \bar{z}+O(2)$ into the differential equation $\dot{z}=X(z, \bar{z})$ (using that $\dot{\varphi}(t)=$ $\left.a_{1,0} \varphi(t)+a_{0,1} \overline{\varphi(t)}+O(2)\right)$, and by a power comparison argument we have

$$
\begin{aligned}
& \dot{\varphi}_{1,0}(t)=a_{1,0} \varphi_{1,0}(t)+a_{0,1} \bar{\varphi}_{0,1}(t), \\
& \dot{\varphi}_{0,1}(t)=a_{0,1} \bar{\varphi}_{1,0}(t)+a_{1,0} \varphi_{0,1}(t) .
\end{aligned}
$$

We solve the linear initial value problem given by the above equations and their conjugates with the initial conditions $\varphi_{1,0}(0)=1$ and $\varphi_{0,1}(0)=0($ since $\varphi(0 ; z, \bar{z})=z)$, and we obtain the solutions $\varphi_{1,0}$ and $\varphi_{0,1}$, that we omit for the sake of shortness. At this stage, by imposing that $\varphi_{1,0}(1)=\mathrm{e}^{i \alpha}$ and $\varphi_{0,1}(1)=0$, we get that $a_{1,0}=i \alpha$ and $a_{0,1}=0$, which proves the result.

Hence, form now on, if we impose that $X$ is polynomial of degree $n$ we can write $X(z, \bar{z})=i \alpha z+\sum_{j+k=2}^{n} a_{j, k} z^{j} \bar{z}^{k}$. When we only assume that it is of class $\mathcal{C}^{n+1}$, near the origin we can write it as $X(z, \bar{z})=i \alpha z+\sum_{j+k=2}^{n} a_{j, k} z^{j} \bar{z}^{k}+O(n+1)$. In any case, by plugging the Taylor expansion of $\varphi(t ; z, \bar{z})$ in the expression of the differential system $\dot{z}=X(z, \bar{z})$, that is by imposing $d \varphi(t ; z, \bar{z}) / d t=X(\varphi(t), \overline{\varphi(t)})=i \alpha \varphi(t ; z, \bar{z})+\sum_{j+k=2}^{n} a_{j, k} \varphi^{j}(t, z, \bar{z}) \bar{\varphi}^{k}(t, z, \bar{z})+$ $O(n+1)$, and from a power comparison argument we get the following result: 
Lemma 4. Let $X(z, \bar{z})=i \alpha z+\sum_{j+k=2}^{n} a_{j, k} z^{j} \bar{z}^{k}+O(n+1)$ with $\alpha \in(0,2 \pi)$ be a planar $\mathcal{C}^{n+1}$ vector field. Then, in a neighborhood of the origin, its flow is given by $\varphi(t ; z, \bar{z})=$ $\mathrm{e}^{i \alpha t} z+\sum_{j+k=2}^{n} \varphi_{j, k}(t) z^{j} \bar{z}^{k}+O(n+1)$, where for each $j, k \in \mathbb{N}$ such that $2 \leq j+k \leq n$ the functions $\varphi_{j, k}(t)$ satisfy the linear differential equation

$$
\dot{\varphi}_{j, k}(t)=i \alpha \varphi_{j, k}(t)+a_{j, k} \mathrm{e}^{i(j-k) \alpha t}+b_{j, k}(t) \text { with } \varphi_{j, k}(0)=0,
$$

where $b_{j, k}(t)=\sum_{\gamma \in S_{j, k}} P_{\gamma}(t) \mathrm{e}^{\gamma i t}$ and $S_{j, k} \subset \mathbb{Z}$ is a finite set, $P_{\gamma}$ depends on the values on the coefficients $a_{\ell, m}$ and the functions $\varphi_{\ell, m}(t)$ with $2 \leq \ell+m<j+k$.

By using the above result, given a map as in (3), we want either to obtain a planar polynomial vector field $X(z, \bar{z})$ such that in a neighborhood of the origin its flow satisfies (5) or to prove that there is no $\mathcal{C}^{n+1}$ vector field whose flow satisfies (5). We do it by a recursive procedure.

Indeed, suppose that we have computed the coefficients of $X$ up to order $\kappa-1$ for $2<\kappa \leq n$. To compute any coefficient $a_{j, k}$ with $j+k=\kappa$, we solve the initial value problem (6) and impose Equation (5). If $j-k-1=0$, then

$$
\varphi_{j, k}(1)=\mathrm{e}^{i \alpha}\left[a_{j, k}+\int_{0}^{1} b_{j, k}(\tau) \mathrm{e}^{-i \alpha \tau} d \tau\right]=f_{j, k} .
$$

In this case we can isolate the coefficient $a_{j, k}$, thus contributing to determinate the expression of the vector field.

If $j-k-1 \neq 0$, then we have

$$
\varphi_{j, k}(1)=\mathrm{e}^{i \alpha}\left[\frac{a_{j, k}}{i(j-k-1) \alpha}\left(\mathrm{e}^{i(j-k-1) \alpha}-1\right)+\int_{0}^{1} b_{j, k}(\tau) \mathrm{e}^{-i \alpha \tau} d \tau\right]=f_{j, k} .
$$

From the above equation we always can isolate the coefficient $a_{j, k}$ except in the case that

$$
\mathrm{e}^{i(j-k-1) \alpha}-1=0
$$

or, in other words if $\mathrm{e}^{i \alpha}$ is a $|j-k-1|$-root of the unity. In this case we say that there appears a resonance associated with the coefficient $a_{j, k}$, and the equation (7) is satisfied for every value of $a_{j, k}$ (thus leading to a parametric family of vector fields) if and only if it is satisfied the compatibility equation corresponding to the coefficient $a_{j, k}$ :

$$
\mathrm{e}^{i \alpha} \int_{0}^{1} b_{j, k}(\tau) \mathrm{e}^{-i \alpha \tau} d \tau=f_{j, k}
$$

Otherwise, we get an obstruction for $F$ to be the time-1 map of a polynomial (or $\mathcal{C}^{n+1}$ ) vector field, see the proof of Theorem 2 for examples of polynomial maps for which there is no vector field whose flow satisfies (5). 
In fact, observe that if for any couple $\ell$ and $m$ with $2 \leq \ell+m<j+k$ there is not a resonance, then the function $b_{j, k}(t)=\sum_{\gamma \in S_{j, k}} P_{\gamma}(t) \mathrm{e}^{\gamma i t}$ introduced in Lemma 4, depends on the values of the previous coefficients $a_{\ell, m}$, thus on the previous coefficients $f_{\ell, m}$. On the contrary, if there exists a couple of values $\ell$ and $m$ with $2 \leq \ell+m<j+k$ giving rise to a resonance (that is, $\mathrm{e}^{i \alpha}$ is a $|\ell-m-1|$-root of the unity) and the compatibility condition associated with $a_{\ell, m}$ is satisfied, then the function $b_{j, k}(t)$ also depends on the parameter $a_{\ell, m}$.

Also observe that a resonance may appear at different order levels, so that in order to obtain the associated vector field, we must identify the first order in which a resonance appears and verify that each compatibility equation is fulfilled. In that case, we can proceed by solving the different equations (6) for higher orders, by carrying the expressions of the indeterminate terms, and verifying that the next different compatibility equations are also satisfied.

Remark 5. Fixing an order $n$, if we consider the pairs $(j, k)$ with $j+k=n$ we get that $|j-k-1| \in\{0,2,4, \ldots, n+1\}$ if $n$ is odd and $|j-k-1| \in\{1,3,5, \ldots, n+1\}$ if $n$ is even. Hence, if a resonance appears at order $n$ and it has not appeared at order $k<n$, then $\mathrm{e}^{i \alpha}$ is an $m$-root of unity with $m \in\{2,4, \ldots, n+1\}$ if $n$ is odd and $m \in\{3,5, \ldots, n+1\}$ if $n$ is even.

Summarizing the above recursive procedure we obtain the following result:

Theorem 6. Consider a polynomial map $F$ of degree $n$ of the form (3).

(i) If $\mathrm{e}^{i \alpha}$ is not $a|j-k-1|$-root of the unity for all couple $j, k$ with $j+k \in\{0,1, \ldots, n\}$ then there exists a unique polynomial vector field of degree at most $n$ such that its associated flow satisfies $\varphi(1 ; z, \bar{z})=F(z, \bar{z})+O(n+1)$.

(ii) If $\mathrm{e}^{i \alpha}$ is $a|j-k-1|$-root of the unity for certain $j, k$ with $j+k \in\{0,1, \ldots, n\}$ and the compatibility equation (8) corresponding to the coefficient $a_{j, k}$ is not satisfied, then there is no $\mathcal{C}^{n+1}$ vector field such that its associated flow satisfies $\varphi(1 ; z, \bar{z})=$ $F(z, \bar{z})+O(n+1)$.

(iii) If there are $\ell$ couples $j, k$ with $j+k \in\{0,1, \ldots, n\}$ such that $\mathrm{e}^{i \alpha}$ is a $|j-k-1|$-root of the unity and the compatibility equations (8) corresponding to the coefficients $a_{j, k}$ are satisfied, then there exists an $\ell$-parametric family of polynomial vector fields of degree at most $n$ satisfying $\varphi(1 ; z, \bar{z})=F(z, \bar{z})+O(n+1)$.

In the next sections we present the explicit expressions for the vector fields associated with quadratic and cubic maps of the form (3), satisfying Equation (5) for $n=2$ and $n=3$ respectively. 


\section{$2.2 \quad$ Vector fields for quadratic maps}

The whole scene in the quadratic case is described in the next proposition. Observe that in the above scheme, at order two a resonance can only occur if $\omega=\mathrm{e}^{i \alpha}$ is a cubic root of unity. This can be seen by taking the function $r(j, k)=|j-k-1|$ and observing that it takes the values $r(2,0)=r(1,1)=1$ and $r(0,2)=3$.

Proposition 7. Set $F(z, \bar{z})=\omega z+\sum_{j+k=2} f_{j, k} z^{j} \bar{z}^{k}$, where $\omega=\mathrm{e}^{i \alpha}$ with $\alpha \in(0,2 \pi)$. Then

(a) If $\omega$ is not a cubic root of unity, then there exists a unique quadratic vector field satisfying (5) with $n=2$, given by $X(z, \bar{z})=i \alpha z+\sum_{j+k=2} a_{j, k} z^{j} \bar{z}^{k}$ where

$$
a_{2,0}=\frac{i \alpha f_{2,0}}{\omega(\omega-1)}, \quad a_{1,1}=\frac{i \alpha f_{1,1}}{\omega-1}, \quad a_{0,2}=\frac{3 i \alpha \omega^{2} f_{0,2}}{\omega^{3}-1} .
$$

(b) Assume that $\omega$ is a cubic root of unity. If $f_{0,2}=0$, then there exists an one-parameter family of quadratic vector fields satisfying (5) with $n=2$. In this case the coefficients $a_{2,0}$ and $a_{1,1}$ of such a vector field are the ones given in Equation (9) and $a_{0,2}$ is the free parameter. If $f_{0,2} \neq 0$ then there is no $\mathcal{C}^{3}$ vector field satisfying (5) with $n=2$.

Proof. Consider a quadratic map $F(z, \bar{z})=\mathrm{e}^{i \alpha} z+f_{2,0} z^{2}+f_{1,1} z \bar{z}+f_{0,2} \bar{z}^{2}$ and a vector field of the form $X(z, \bar{z})=i \alpha z+a_{2,0} z^{2}+a_{1,1} z \bar{z}+a_{0,2} \bar{z}^{2}$. If we search for its associated flow $\varphi(t ; z, \bar{z})=\mathrm{e}^{i \alpha t} z+\varphi_{2,0}(t) z^{2}+\varphi_{1,1}(t) z \bar{z}+\varphi_{0,2}(t) \bar{z}^{2}+O(3)$, by plugging this expression in the differential equation $\dot{z}=X(z, \bar{z})$, we get that

$$
\begin{gathered}
\dot{z}=i \alpha \mathrm{e}^{i \alpha t} z+\dot{\varphi}_{2,0}(t) z^{2}+\dot{\varphi}_{1,1}(t) z \bar{z}+\dot{\varphi}_{0,2}(t) \bar{z}^{2}+O(3)=X(z, \bar{z})= \\
=i \alpha \mathrm{e}^{i \alpha t} z+\left(i \alpha \varphi_{2,0}(t)+a_{2,0} \mathrm{e}^{2 i \alpha t}\right) z^{2}+\left(i \alpha \varphi_{1,1}(t)+a_{1,1}\right) z \bar{z}+\left(i \alpha \varphi_{0,2}(t)+a_{0,2} \mathrm{e}^{-2 i \alpha t}\right) \bar{z}^{2}+O(3),
\end{gathered}
$$

so we obtain the differential equations:

$$
\begin{aligned}
& \dot{\varphi}_{2,0}(t)=i \alpha \varphi_{2,0}(t)+a_{2,0} \mathrm{e}^{2 i \alpha t}, \\
& \dot{\varphi}_{1,1}(t)=i \alpha \varphi_{1,1}(t)+a_{1,1}, \\
& \dot{\varphi}_{0,2}(t)=i \alpha \varphi_{0,2}(t)+a_{0,2} \mathrm{e}^{-2 i \alpha t} .
\end{aligned}
$$

with the conditions $\varphi_{2,0}(0)=0, \varphi_{1,1}(0)=0$ and $\varphi_{0,2}(0)=0$ (remember that $\left.\varphi(0 ; z, \bar{z})=z\right)$. By integrating them, evaluating their solutions at time $t=1$ and imposing $\varphi(1 ; z, \bar{z})=$ $F(z, \bar{z})+O(3)$ we get the corresponding equations (6):

$$
\begin{aligned}
\varphi_{2,0}(1) & =\frac{i}{\alpha} a_{2,0}\left(1-\mathrm{e}^{i \alpha}\right) \mathrm{e}^{i \alpha}=f_{2,0}, \\
\varphi_{1,1}(1) & =\frac{i}{\alpha} a_{1,1}\left(1-\mathrm{e}^{i \alpha}\right)=f_{1,1}, \\
\varphi_{0,2}(1) & =-\frac{i}{3 \alpha} a_{0,2}\left(1-\mathrm{e}^{-3 i \alpha}\right) \mathrm{e}^{i \alpha}=f_{0,2} .
\end{aligned}
$$


Since $\alpha \in(0,2 \pi)$, the first two equations can always be solved giving the values for $a_{2,0}$ and $a_{1,1}$ in Equation (9). The third equation fixes a value of $a_{0,2}$ unless $\omega$ is a third root of unity, obtaining the expressions in (9), thus proving $(a)$.

If $\omega$ is a cubic root of the unity, then the compatibility condition (8) associated with the coefficient $a_{0,2}$ is $f_{0,2}=0$, and the result in statement $(b)$ follows.

\subsection{Vector fields for cubic maps}

Given a cubic map, to search a cubic vector field satisfying (5) with $n=3$, first we notice that the resonances only occur when $\mathrm{e}^{i \alpha}$ is a third root of the unity, when is a square root of the unity, or when is a primitive fourth root of the unity, see Remark 5. According to Theorem 6 , if $\mathrm{e}^{i \alpha}$ is not such a root of the unity there exists a unique polynomial vector field satisfying (5).

Also according to Theorem 6 , if $\mathrm{e}^{i \alpha}$ is a third root of the unity and the compatibility condition associated to $a_{0,2}$ is satisfied, then there exists an one-parameter family of vector fields satisfying (5). If $\mathrm{e}^{i \alpha}$ is a square root of the unity (hence it also is a fourth-root of unity) and the compatibility condition associated with $a_{3,0}, a_{1,2}$ and $a_{0,3}$ are fulfilled, then there exists a three-parametric family of such vector fields. And finally, if $\mathrm{e}^{i \alpha}$ is a primitive quartic root of the unity and the compatibility condition associated with $a_{0,3}$ holds, then there exists an one-parameter family of such vector fields. All these four cases are studied in the next four propositions:

Proposition 8. Set $F(z, \bar{z})=\omega z+\sum_{j+k=2}^{3} f_{j, k} z^{j} \bar{z}^{k}$, where $\omega=\mathrm{e}^{i \alpha}$ with $\alpha \in(0,2 \pi)$. If $\omega$ is not a quadratic, cubic or fourth root of unity, then there exists a unique cubic vector field satisfying (5) with $n=3, X(z, \bar{z})=i \alpha z+\sum_{j+k=2}^{3} a_{j, k} z^{j} \bar{z}^{k}$, where the coefficients $a_{2,0}, a_{1,1}$ and $a_{0,2}$ are the ones given in (9), and

$$
a_{3,0}=\frac{-i \alpha P_{3,0}}{\omega^{2}\left(\omega^{3}-1\right)(\omega+1)},
$$

with

$$
\begin{gathered}
P_{3,0}=\left(\overline{f_{0,2}} f_{1,1}-2 f_{3,0}\right) \omega^{3}+2\left(\overline{f_{0,2}} f_{1,1}+f_{2,0}^{2}-f_{3,0}\right) \omega^{2}+2\left(f_{2,0}^{2}-f_{3,0}\right) \omega+2 f_{2,0}^{2} \\
a_{2,1}=\frac{-i P_{2,1}}{\omega^{2}\left(\omega^{3}-1\right)^{2}}
\end{gathered}
$$


with

$$
\begin{aligned}
P_{2,1}= & \left((i+\alpha)\left|f_{1,1}\right|^{2}+i f_{2,1}\right) \omega^{7}+\left((i+2 \alpha)\left|f_{1,1}\right|^{2}-2 i f_{1,1} f_{2,0}\right) \omega^{6}+ \\
& \left((i+3 \alpha)\left|f_{1,1}\right|^{2}+(2 i+6 \alpha)\left|f_{0,2}\right|^{2}-f_{1,1} f_{2,0}(i+\alpha)\right) \omega^{5}+ \\
& \left((-i+2 \alpha)\left|f_{1,1}\right|^{2}-(i+2 \alpha) f_{2,0} f_{1,1}-2 i f_{2,1}\right) \omega^{4}+\left(3 f_{1,1} f_{2,0}-\left|f_{1,1}\right|^{2}\right) \times \\
& (i-\alpha) \omega^{3}+\left(-i\left|f_{1,1}\right|^{2}-2 i\left|f_{0,2}\right|^{2}+(i-2 \alpha) f_{1,1} f_{2,0}\right) \omega^{2}+ \\
& \left(f_{2,0} f_{1,1}(i-\alpha)+i f_{2,1}\right) \omega-i f_{1,1} f_{2,0}
\end{aligned}
$$

$$
a_{1,2}=\frac{-i \alpha P_{1,2}}{\left(\omega^{3}-1\right)(\omega+1)}
$$

with

$$
\begin{aligned}
P_{1,2}= & f_{1,1} \overline{f_{2,0}} \omega^{4}+\left(2 \overline{f_{1,1}} f_{0,2}+\overline{f_{2,0}} f_{1,1}-2 f_{1,2}\right) \omega^{3}+\left(4 \overline{f_{1,1}} f_{0,2}+\overline{f_{2,0}} f_{1,1}+4 f_{0,2} f_{2,0}+\right. \\
& \left.f_{1,1}^{2}-2 f_{1,2}\right) \omega^{2}+\left(2 f_{0,2} f_{2,0}+f_{1,1}^{2}-2 f_{1,2}\right) \omega+f_{1,1}^{2}
\end{aligned}
$$

and

$$
a_{0,3}=\frac{-i \alpha \omega^{2} P_{0,3}}{\left(\omega^{2}+1\right)\left(\omega^{3}-1\right)(\omega+1)},
$$

with

$$
\begin{aligned}
P_{0,3}= & 2 f_{0,2} \overline{f_{2,0}} \omega^{4}+4\left(f_{0,2} \overline{f_{2,0}}-f_{0,3}\right) \omega^{3}+\left(6 f_{0,2} \overline{f_{2,0}}+3 f_{0,2} f_{1,1}-4 f_{0,3}\right) \omega^{2}+ \\
& 2\left(f_{0,2} f_{1,1}-2 f_{0,3}\right) \omega+f_{0,2} f_{1,1} .
\end{aligned}
$$

Proof. Consider the cubic map $F(z, \bar{z})$ and a cubic vector field $X(z, \bar{z})$. We follow the recursive procedure based on Lemma 4 . Observe that the coefficients $a_{3,0}, a_{2,1}, a_{1,2}$ and $a_{0,3}$ will depend on the expressions of the quadratic coefficients $a_{2,0}, a_{1,1}$, and $a_{0,2}$ that must be computed firstly. We plug the expression $\varphi(t ; z, \bar{z})=\mathrm{e}^{i \alpha} z+\sum_{j+k=2}^{3} \varphi_{j, k}(t) z^{j} \bar{z}^{k}+O(4)$ into the differential equation $\dot{z}=X(z, \bar{z})$, getting the equation

$$
i \alpha \mathrm{e}^{i \alpha t} z+\sum_{j+k=2}^{3} \dot{\varphi}_{j, k}(t) z^{j} \bar{z}^{k}+O(4)=i \alpha \mathrm{e}^{i \alpha t} z+\sum_{j+k=2}^{3} R_{j, k}(t) z^{j} \bar{z}^{k}+O(4),
$$

where each term $R_{j, k}(t)$ has the form of the right-hand side of equation (6). By a simple power comparison argument we arrive to each equation (6) for $j+k=2,3$.

The equations corresponding to the quadratic terms are obtained by repeating the steps proof of Proposition 7. After this steps we obtain the quadratic coefficients given in (9) and also the functions $\varphi_{2,0}, \varphi_{1,1}$ and $\varphi_{0,2}$, in terms of the quadratic coefficients of the map $f_{2,0}, f_{1,1}$ and $f_{0,2}$. 
For the cubic terms we follow the same procedure. To obtain the coefficient $a_{3,0}$ we equal the terms with $z^{3}$ in both sides of equation (11), and we get

$$
\dot{\varphi}_{3,0}(t)=i \alpha \varphi_{3,0}(t)+a_{3,0} \mathrm{e}^{3 i \alpha t}+\frac{i \alpha Q_{3,0}(t)}{\omega^{2}(\omega-1)^{2}\left(\omega^{2}+\omega+1\right)},
$$

where

$$
Q_{3,0}(t)=-2\left(\omega^{2}+\omega+1\right) f_{2,0}^{2} \mathrm{e}^{2 i \alpha t}+\left(\overline{f_{0,2}} f_{1,1} \omega^{3}+2\left(\omega^{2}+\omega+1\right) f_{2,0}^{2}\right) \mathrm{e}^{3 i \alpha t}-\overline{f_{0,2}} f_{1,1} \omega^{3} .
$$

By integrating this differential equation and imposing Equation (5), we obtain the corresponding Equation (7):

$$
\frac{-i\left(\omega^{2}-1\right) \omega a_{3,0}}{2 \alpha}+\frac{\overline{f_{0,2}} f_{1,1} \omega^{3}+\left(2 \overline{f_{0,2}} f_{1,1}+2 f_{2,0}^{2}\right) \omega^{2}+2 f_{2,0}^{2} \omega+2 f_{2,0}^{2}}{2 \omega\left(\omega^{2}+\omega+1\right)}=f_{3,0},
$$

thus we get the expression of the coefficient $a_{3,0}$ in the statement.

The expressions of the rest of cubic coefficients are obtained in an analogous way.

Proposition 9. Set $F(z, \bar{z})=\omega z+\sum_{j+k=2}^{3} f_{j, k} z^{j} \bar{z}^{k}$, where $\omega=\mathrm{e}^{i \alpha}$ is a primitive third root of unity. Then there exists a cubic vector field $X$ satisfying (5) for $n=3$ if and only if $f_{0,2}=0$. In this case, there is an one-parameter family of cubic vector fields $X$ satisfying (5) for $n=3$, whose coefficients $a_{2,0}$ and $a_{1,1}$ are the ones given in Equation (9), $a_{0,2}$ is a free parameter, and

$$
a_{3,0}=\frac{\overline{a_{0,2}} f_{1,1}(\omega-1)^{2}-6 i \alpha f_{3,0} \omega+6 i \alpha f_{2,0}^{2}}{3 \omega(\omega-1)}, \quad a_{2,1}=\frac{i P_{2,1}}{3 \alpha(\omega-1)^{2}},
$$

with

$$
\begin{aligned}
& P_{2,1}=\left(9 i \alpha f_{1,1} f_{2,0}-3 f_{2,0} f_{1,1} \alpha^{2}+3 i \alpha f_{2,1}-2\left(\left|a_{0,2}\right|\right)^{2}\right) \omega^{2} \\
&+\left(3 i\left(\left|f_{1,1}\right|\right)^{2} \alpha+3\left(\left|f_{1,1}\right|\right)^{2} \alpha^{2}-3 i \alpha f_{1,1} f_{2,0}+3 i \alpha f_{2,1}+4\left(\left|a_{0,2}\right|\right)^{2}\right) \omega \\
&-3 i\left(\left|f_{1,1}\right|\right)^{2} \alpha-6 i \alpha f_{1,1} f_{2,0}-6 i \alpha f_{2,1}-2\left(\left|a_{0,2}\right|\right)^{2} ; \\
& a_{1,2}=\frac{i P_{1,2}}{3(1-\omega)},
\end{aligned}
$$

with

$$
\begin{aligned}
P_{1,2}= & \left(-6 i f_{1,2} \alpha+2 \overline{f_{1,1}} a_{0,2}+4 f_{2,0} a_{0,2}\right) \omega^{2}+i\left(3 i \alpha f_{1,1}^{2}-4 \overline{f_{1,1}} a_{0,2}-2 f_{2,0} a_{0,2}\right) \omega \\
& +i\left(3 i \overline{f_{2,0}} \alpha f_{1,1}+2 \overline{f_{1,1}} a_{0,2}-2 f_{2,0} a_{0,2}\right) ;
\end{aligned}
$$

and

$$
a_{0,3}=\frac{P_{0,3}}{3(1-\omega)}
$$

with

$$
P_{0,3}=-\left(6 a_{0,2} \overline{f_{2,0}}+f_{1,1} a_{0,2}\right) \omega^{2}+\left(2 a_{0,2} \overline{f_{2,0}}-f_{1,1} a_{0,2}\right) \omega-12 i f_{0,3} \alpha+4 a_{0,2} \overline{f_{2,0}}+2 f_{1,1} a_{0,2} .
$$


Proof. We follow the same steps as in the proof of Proposition 8, but we take into account that as mentioned before, and as can be seen in the proof of Proposition 7, when $\mathrm{e}^{i \alpha}$ is a third root of the unity, the only compatibility condition that appears is the one associated with the coefficient $a_{0,2}$, and it is $f_{0,2}=0$. Assuming now this condition, we obtain a new Equation (11). Setting $a_{0,2}$ as a free parameter and fixing the values of the coefficients $a_{2,0}$ and $a_{1,1}$ as the ones in Equation (9), we proceed to compute the coefficients of the cubic term. To obtain $a_{3,0}$, we equal the terms with $z^{3}$ in both sides of the corresponding equation (11), and we get

$$
\dot{\varphi}_{3,0}(t)=i \alpha \varphi_{3,0}(t)+a_{3,0} \mathrm{e}^{3 i \alpha t}+\frac{\widetilde{Q}_{3,0}(t)}{3 \omega^{2}(\omega-1)^{2}},
$$

where

$$
\widetilde{Q}_{3,0}(t)=-6 i \alpha f_{2,0}^{2} \mathrm{e}^{2 i \alpha t}+\left(-\overline{a_{0,2}} f_{1,1} \omega^{2}+6 i \alpha f_{2,0}^{2}+\overline{a_{0,2}} f_{1,1}\right) \mathrm{e}^{3 i \alpha t}+\overline{a_{0,2}} f_{1,1}\left(\omega^{2}-1\right) .
$$

By integrating this equation, imposing Equation (5) and taking into account that $\omega^{3}=1$, we get that the corresponding Equation (7) is:

$$
\frac{i(\omega-1) a_{3,0}}{2 \alpha}-\frac{i\left(\overline{a_{0,2}} f_{1,1}(\omega-1)^{2}+6 i \alpha f_{2,0}^{2}\right)}{6 \omega \alpha}=f_{3,0} .
$$

Thus we get the expression of the coefficient $a_{3,0}$ in the statement. The expressions of the other coefficients are obtained similarly.

If $\omega=\mathrm{e}^{i \alpha}$ is a squared root of the unity, then $\alpha=\pi$ (since $\alpha \neq 0$ ). In this case the compatibility conditions (8) are the ones associated with the coefficients $a_{3,0}$ and $a_{1,2}$ but also $a_{0,3}$, because $\omega^{2}=1$ implies $\omega^{4}=1$. Proceeding as in the previous results, we obtain:

Proposition 10. Set $F(z, \bar{z})=-z+\sum_{j+k=2}^{3} f_{j, k} z^{j} \bar{z}^{k}$. Then there exists a cubic vector field $X(z, \bar{z})=i \pi z+\sum_{j+k=2}^{3} a_{j, k} z^{j} \bar{z}^{k}$ satisfying (5) for $n=3$, if and only if

$$
\begin{aligned}
f_{3,0} & =-\frac{1}{2} f_{1,1} \overline{f_{0,2}}-f_{2,0}^{2}, \\
f_{1,2} & =-\frac{1}{2} f_{1,1}^{2}-\frac{1}{2} f_{1,1} \overline{f_{0,2}}-f_{2,0} f_{0,2}-f_{0,2} \overline{f_{1,1}}, \\
f_{0,3} & =-\frac{1}{2} f_{0,2}\left(2 \overline{f_{2,0}}+f_{1,1}\right) .
\end{aligned}
$$

If these equations are fulfilled, then there is a three-parameter family of cubic vector fields satisfying (5) for $n=3$, and it is given by

$$
\begin{gathered}
a_{2,0}=\frac{\pi i}{2} f_{2,0}, \quad a_{1,1}=-\frac{\pi i}{2} f_{1,1}, \quad a_{0,2}=-\frac{3 \pi i}{2} f_{0,2}, \\
a_{2,1}=\frac{1}{4}(i \pi-2)\left|f_{1,1}\right|^{2}+\frac{1}{2}(3 i \pi-2)\left|f_{0,2}\right|^{2}-\left(\frac{3}{2}+\frac{\pi i}{4}\right) f_{2,0} f_{1,1}-f_{2,1},
\end{gathered}
$$

and $a_{3,0}, a_{1,2}$ and $a_{0,3}$ are free parameters. 
The resonant case that appears when $\omega=\mathrm{e}^{i \alpha}$ is a primitive fourth root of the unity is studied in the following result:

Proposition 11. Set $F(z, \bar{z})=\omega z+\sum_{j+k=2}^{3} f_{j, k} z^{j} \bar{z}^{k}$. Then,

(a) If $\omega=i$ (that is $\alpha=\frac{\pi}{2}$ ), then there exists a cubic vector field $X(z, \bar{z})=i \frac{\pi}{2} z+$ $\sum_{j+k=2}^{3} a_{j, k} z^{j} \bar{z}^{k}$ satisfying (5) for $n=3$, if and only if

$$
f_{0,3}=\frac{1}{2} f_{0,2}\left((2+2 i) \overline{f_{2,0}}+(1-i) f_{1,1}\right) .
$$

If this equation is fulfilled, then there is an one-parameter family of vector fields satisfying (5) for $n=3$, given by

$$
a_{2,0}=-\frac{\pi}{4}(1+i) f_{2,0}, \quad a_{1,1}=\frac{\pi}{4}(1-i) f_{1,1}, \quad a_{0,2}=\frac{3 \pi}{4}(1+i) f_{0,2}
$$

and

$$
\begin{aligned}
a_{3,0}= & -\frac{\pi}{2}\left(-\left(1+\frac{i}{2}\right) f_{1,1} \overline{f_{0,2}}+i f_{2,0}^{2}+f_{3,0}\right), \\
a_{2,1}= & \frac{1}{4}(-2+(\pi-2) i)\left|f_{1,1}\right|^{2}+\frac{1}{2}(-2+(3 \pi+2) i)\left|f_{0,2}\right|^{2} \\
& +\frac{1}{4}(6+(\pi-2) i) f_{1,1} f_{2,0}-i f_{2,1}, \\
a_{1,2}= & \frac{\pi}{2}\left(-(2+i) f_{0,2} \overline{f_{1,1}}-\frac{i}{2} f_{1,1} \overline{f_{2,0}}-(2-i) f_{2,0} f_{0,2}+\frac{i}{2} f_{1,1}^{2}+f_{1,2}\right),
\end{aligned}
$$

being $a_{0,3}$ the free parameter.

(b) If $\omega=-i$ (that is $\alpha=\frac{3 \pi}{2}$ ), then there exists a cubic vector field $X(z, \bar{z})=i \frac{3 \pi}{2} z+$ $\sum_{j+k=2}^{3} a_{j, k} z^{j} \bar{z}^{k}$ satisfying (5) for $n=3$, if and only if

$$
f_{0,3}=\frac{1}{2} f_{0,2}\left((1+i) f_{1,1}+(2-2 i) \overline{f_{2,0}}\right) .
$$

If this equation is fulfilled, then the there is an one-parameter family of cubic vector fields satisfying (5) for $n=3$, and it is given by

$$
a_{2,0}=\frac{3 \pi}{4}(1-i) f_{2,0}, \quad a_{1,1}=-\frac{3 \pi}{4}(1+i) f_{1,1}, \quad a_{0,2}=\frac{9 \pi}{4}(-1+i) f_{0,2}
$$

and

$$
\begin{aligned}
a_{3,0}= & -\frac{3 \pi}{2}\left(\left(1-\frac{i}{2}\right) f_{1,1} \overline{f_{0,2}}+i f_{2,0}^{2}-f_{3,0}\right), \\
a_{2,1}= & \frac{1}{4}(-2+(3 \pi+2) i)\left|f_{1,1}\right|^{2}+\frac{1}{2}(-2+(9 \pi-2) i)\left|f_{0,2}\right|^{2} \\
& +\left(\frac{3}{2}+i\left(\frac{1}{2}+\frac{3}{4} \pi\right)\right) f_{2,0} f_{1,1}+i f_{2,1}, \\
a_{1,2}= & \frac{3 \pi}{2}\left((2-i) f_{0,2} \overline{f_{1,1}}-\frac{i}{2} \overline{f_{2,0}} f_{1,1}+(2+i) f_{2,0} f_{0,2}+\frac{i}{2} f_{1,1}{ }^{2}-f_{1,2}\right),
\end{aligned}
$$

being $a_{0,3}$ the free parameter. 


\section{Proof of Theorem 1}

Theorem 1 is a consequence of the following two results. The first one is proved in [8] but for completeness we include a sketch of its proof. The second one is a consequence of the results in the previous section.

Proposition 12. The two polynomial maps

$$
F_{1}(z, \bar{z})=i z+(1-3 i) z^{2}+z \bar{z} \quad \text { and } \quad F_{2}(z, \bar{z})=\frac{1}{2}(1+i \sqrt{3}) z-z^{2} \bar{z},
$$

have the origin as a LAS fixed point for both of them, while the composition map $F_{2} \circ F_{1}$ has the origin as a repeller fixed point.

We notice that the maps $F_{1}$ and $F_{2}$ in the above result are the complex representation of the real maps

$$
\begin{aligned}
& f_{1}(x, y)=\left(-y+2 x^{2}+6 x y, x-3 x^{2}+2 x y+3 y^{2}\right) \\
& f_{2}(x, y)=\left(\frac{x}{2}-\frac{\sqrt{3}}{2} y-x\left(x^{2}+y^{2}\right), \frac{\sqrt{3}}{2} x+\frac{1}{2} y-y\left(x^{2}+y^{2}\right)\right)
\end{aligned}
$$

introduced in [8, Example 7].

Proof of Proposition 12. Let $\mathcal{U}$ be a small enough neighborhood of the origin. Recall that for a suitable $m \in \mathbb{N} \cup\{0\}$, a $\mathcal{C}^{2 m+2}$ map $F$ in $\mathcal{U}$ with an elliptic fixed point whose eigenvalues $\lambda, \bar{\lambda}=1 / \lambda$, are not roots of unity of order $\ell$ for $0<\ell \leq 2 m+1$, is locally conjugate to its Birkhoff normal form:

$$
F_{B}(z, \bar{z})=\lambda z\left(1+\sum_{j=1}^{m} B_{j}(z \bar{z})^{j}\right)+O(2 m+2),
$$

see [1]. The first non-vanishing number $B_{j}$ is called the $j$ th Birkhoff constant. If $V_{j}=$ $\operatorname{Re}\left(B_{j}\right)<0$ (resp. $V_{j}>0$ ), then the point $p$ is LAS (resp. repeller), see [8, Lem. 4.1] for instance. The quantity $V_{j}$ is called the $j$ th Birkhoff stability constant. This is so, because the fact that $V_{j} \neq 0$ implies that the function $z \bar{z}=|z|^{2}$ is a strict Lyapunov function at the origin for the normal form map $F_{B}$ of $F$.

In [8], both the Birkhoff and the Birkhoff stability constants of $F_{1}$ and $F_{2}$ are computed obtaining that $B_{1}\left(F_{1}\right)=-\frac{1}{2}-\frac{11}{2} i$ and $B_{1}\left(F_{2}\right)=-\frac{1}{2}+\frac{\sqrt{3}}{2} i$. So $V_{1}\left(F_{j}\right)=-\frac{1}{2}<0$ for $j=1,2$, and the origin is LAS for both maps $F_{1}$ and $F_{2}$. Also in this reference it is proved that $V_{1}\left(F_{2} \circ F_{1}\right)=\frac{1}{2}(3 \sqrt{3}-5)>0$, so that the origin is a repeller fixed point for the composition map. 
Proposition 13. Consider the planar polynomial vector fields

$$
\begin{aligned}
X_{1}(z, \bar{z}, \mu)= & \frac{i \pi}{2} z-\left(1-\frac{i}{2}\right) \pi z^{2}+\left(\frac{1}{4}-\frac{i}{4}\right) \pi z \bar{z}-(3-4 i) \pi z^{3} \\
& +\left(\frac{3 \pi}{4}-\frac{1}{2}+i\left(\frac{\pi}{2}-\frac{11}{2}\right)\right) z^{2} \bar{z}+\frac{3 \pi}{4} z \bar{z}^{2}+\mu \bar{z}^{3},
\end{aligned}
$$

where $\mu$ is a free parameter, and

$$
X_{2}(z, \bar{z})=\frac{i \pi}{3} z+\left(-\frac{1}{2}+\frac{i \sqrt{3}}{2}\right) z^{2} \bar{z} .
$$

Let $\varphi_{j}(t ; z, \bar{z}), j=1,2$ be their respective associated flows. Then, for $z$ in a small enough neighborhood of the origin

$$
\varphi_{j}(1 ; z, \bar{z})=F_{j}(z, \bar{z})+O(4), \quad j=1,2
$$

where the maps $F_{j}$ are given in Proposition 12.

Proof. Observe that $F_{1}$ has the form (3) with $\alpha=\pi / 2$, so that $\mathrm{e}^{i \alpha}$ is a primitive fourth root of the unity. Since the compatibility condition (12) is satisfied, by using the expression in Proposition $11(a)$ we can find an one-parameter family of vector fields $X_{1}(z, \bar{z}, \mu)$ satisfying $\varphi_{1}(1 ; z, \bar{z})=F_{1}(z, \bar{z})+O(4)$. This is the family of vector fields $X_{1}$ given in the statement, where $\mu$ is the free parameter $a_{0,3}$. Also observe that $F_{2}$ has also the form (3) with $\alpha=\pi / 3$, so that $\mathrm{e}^{i \alpha}$ is a primitive sixth root of the unity. By using Proposition 8 we can find a unique vector field $X_{2}$ satisfying $\varphi_{2}(z, \bar{z})=F_{2}(z, \bar{z})+O(4)$. This $X_{2}$ is the second vector field given in the statement.

Proof of Theorem 1. We will prove that the vector fields given in the statement of Proposition 13 provide the desired example with $X_{1}$ and $X_{2}$ having the origin as a singular LAS point and with the origin being a repeller for the 2 -seasonal differential system (2). Then, the converse situation will hold simply by considering the vector fields $-X_{1}$ and $-X_{2}$.

The key point is to realize that if $\varphi(t ; z, \bar{z})$ denotes the flow of $(2)$ it holds that

$$
\begin{aligned}
\varphi(2 ; z, \bar{z}) & =\varphi_{2}\left(1 ; \varphi_{1}(1 ; z, \bar{z}), \overline{\varphi_{1}(1 ; z, \bar{z})}\right)=F_{2}\left(F_{1}(z, \bar{z})+O(4)\right)+O(4) \\
& =F_{2} \circ F_{1}(z, \bar{z})+O(4) .
\end{aligned}
$$

Now, a crucial step is that the first Birkhoff stability constant $V_{1}(F)$ only depends on the third order jet of $F$ at the fixed point, see [8, Equation (3)]. Hence $V_{1}\left(\varphi_{j}(1 ; z, \bar{z})\right)=V_{1}\left(F_{j}\right)$, $j=1,2$ and $V_{1}(\varphi(2 ; z, \bar{z}))=V_{1}\left(F_{2} \circ F_{1}\right)$.

It is clear that for the vector fields $X_{1}, X_{2}$ and the one in (2) the stability of the origin coincides with the one of the corresponding flows $\varphi_{1}(1 ; \cdot, \cdot), \varphi_{2}(1 ; \cdot, \cdot)$ and $\varphi(2 ; \cdot, \cdot)$ 
respectively. Equivalently, these stabilities coincide with the ones of the origin for the maps $F_{1}, F_{2}$ and $F_{2} \circ F_{1}$. Since, by Proposition 12 , these maps provide a discrete dynamic Parrondo's paradox, we have that both $X_{1}$ and $X_{2}$ have a LAS singular point at the origin, and the corresponding 2-seasonal system (2) has a repeller point at the origin, as we wanted to prove.

\section{Proof of Theorem 2}

(i) This is a corollary of statement $(i)$ of Theorem 6 .

(ii) We will use item (ii) of Theorem 6 . For each $n \geq 2$ we will prove that the polynomial map

$$
F(z, \bar{z})=\mathrm{e}^{i \alpha} z+\bar{z}^{n}, \text { with } \alpha=\frac{2 \pi}{n+1},
$$

satisfies the statement of the theorem.

The result for $n=2$ is a direct consequence of Proposition 7. When $n=3$, the result follows by item (a) of Proposition 11 because the compatibility condition (12) does not hold.

Now suppose that $n \geq 4$. We claim that for each $2 \leq m \leq n-1$, if $\mathrm{e}^{i \alpha}$ is a primitive $(n+1)$-root of unity and $X_{m}$ is a vector field with associated flow of the form $\varphi_{m}(t ; z, \bar{z})=$ $\mathrm{e}^{i \alpha t} z+O(m+1)$, then it satisfies $X_{m}(z, \bar{z})=i \alpha z+O(m+1)$. We will prove the claim by induction on $m$, by using the same method and notations introduced in Section 2.1.

By Proposition 7 the result is true for $m=2$. Assume that the result is true for $m<n-1$. As a consequence, for any vector field of the form

$$
X_{m+1}(z, \bar{z})=i \alpha z+\sum_{j+k=m+1} a_{j, k} z^{j} \bar{z}^{k}+O(m+2),
$$

its associated flow has the form

$$
\varphi_{m+1}(t ; z, \bar{z})=\mathrm{e}^{i \alpha t} z+\sum_{j+k=m+1} \varphi_{j, k}(t) z^{j} \bar{z}^{k}+O(m+2) .
$$

By plugging the above expression into the differential system $\dot{z}=X_{m+1}(z, \bar{z})$, we get that for $j+k=m+1$ :

$$
\varphi_{j, k}^{\prime}(t)=i \alpha \varphi_{j, k}(t)+a_{j, k} \mathrm{e}^{(j-k) i \alpha t},
$$

and since $\varphi_{j, k}(0)=0$ we obtain that

$$
\varphi_{j, k}(t)= \begin{cases}\frac{a_{j, k}}{(j-k-1) i \alpha} \mathrm{e}^{i \alpha t}\left(\mathrm{e}^{(j-k-1) i \alpha t}-1\right), & j \neq k+1, \\ a_{j, k} t \mathrm{e}^{i \alpha t}, & j=k+1 .\end{cases}
$$


Since $\mathrm{e}^{i \alpha}$ is a primitive $(n+1)$-root of unity, $\mathrm{e}^{(j-k-1) i \alpha} \neq 1$ for $j+k=m+1<n$ (see Remark 5). Now, if we assume that $\varphi_{m+1}(1 ; z, \bar{z})=\mathrm{e}^{i \alpha} z+O(m+1)$ then $\varphi_{j, k}(1)=0$ for $j+k=m+1$ and from (15) $a_{j, k}=0$ and $\varphi_{j, k}(t) \equiv 0$. So, the claim is proved.

Now we proceed by contradiction. We consider the map (13), and suppose that there exists a vector field $X$ whose flow satisfies

$$
\varphi(1 ; z, \bar{z})=F(z, \bar{z})+O(n+1)=\mathrm{e}^{i \alpha} z+\bar{z}^{n}+O(n+1) .
$$

From the claim, $X$ must have the form $X(z, \bar{z})=i \alpha z+\sum_{j+k=n} a_{j, k} z^{j} \bar{z}^{k}+O(n+1)$. For these kind of vector fields the associated flow must have the form $\varphi(t ; z, \bar{z})=\mathrm{e}^{i \alpha t}+$ $\sum_{j+k=n} \varphi_{j, k}(t) z^{j} \bar{z}^{k}+O(n+1)$. For $j+k=n$, the functions $\varphi_{j, k}(t)$ also satisfy (14) and hence (15). In particular,

$$
\varphi_{0, n}(t)=-\frac{1}{2 \pi i} a_{0, n} \mathrm{e}^{\frac{2 \pi i}{n+1} t}\left(\mathrm{e}^{-2 \pi i t}-1\right),
$$

and therefore $\varphi_{0, n}(1)=0$. But this is in contradiction with Equation (16), which implies that $\varphi_{0, n}(1)=1$.

\section{Funding}

The authors are supported by Ministry of Economy, Industry and Competitiveness-State Research Agency of the Spanish Government through grants MTM2016-77278-P (MINECO/AEI/FEDER, UE, first and second authors) and DPI2016-77407-P (MINECO/AEI/FEDER, UE, third author). The first and second authors are also supported by the grant 2017-SGR-1617 from AGAUR, Generalitat de Catalunya. The third author acknowledges the group's research recognition 2017-SGR-388 from AGAUR, Generalitat de Catalunya.

\section{Conflict of Interest}

The authors declare that they have no conflict of interest.

\section{References}

[1] D.K. Arrowsmith, C.M. Place. An introduction to dynamical systems. Cambridge University Press, Cambridge 1990.

[2] H. Bass, G. Meisters. Polynomial flows in the plane. Advances in Mathematics 55 (1985), 173-208.

[3] B. Buonomo. N. Chitnis, A. d'Onofrio. Seasonality in epidemic models: a literature review. Ricerche di Matematica 67 (2018), 7-25. 
[4] J.S. Cánovas, A. Linero, D. Peralta-Salas. Dynamic Parrondo's paradox. Physica D 218 (2006), 177-184.

[5] B. Cid, F.M. Hilker, E. Liz. Harvest timing and its population dynamic consequences in a discrete single-species model. Mathematical Biosciences 248 (2014), 78-87.

[6] A. Cima, A. Gasull, V. Mañosa. Non-autonomous 2-periodic Gumovski-Mira difference equations. International Journal of Bifurcations and Chaos in Applied Sciences and Engineering 22 (2012), Paper No 1250264 (14 pages).

[7] A. Cima, A. Gasull, V. Mañosa. Integrability and non-integrability of periodic nonautonomous Lyness recurrences. Dynamical Systems 28 (2013), 518-538.

[8] A. Cima, A. Gasull, V. Mañosa. Parrondo's dynamic paradox for the stability of non-hyperbolic fixed points. Discrete and Continuous Dynamical Systems -series A 38 (2018), 889-904.

[9] S.D. Fretwell. Populations in a seasonal environment. Princeton University Press, Princeton 1972.

[10] J. Guckenheimer, P. Holmes. Nonlinear oscillations, dynamical systems, and bifurcations of vector fields. Springer-Verlag, New York 1990.

[11] G.P. Harmer and D. Abbott. Losing strategies can win by Parrondo's paradox. Nature (London), Vol. 402, No. 6764 (1999), 864.

[12] S.B. Hsu, X.Q. Zhao. A Lotka-Volterra competition model with seasonal succession. Journal of Mathematical Biology 64 (2012), 109-130.

[13] H. Ji, M. Strugarek. Sharp seasonal threshold property for cooperative population dynamics with concave nonlinearities. Bulletin des Sciences Mathématiques 147 (2018), $58-82$.

[14] M. Kot, W.M. Schaffer. The effects of seasonality on discrete models of population growth. Theoretical Population Biology 26 (1984), 340-360.

[15] E. Liz. Effects of strength and timing of harvest on seasonal population models: stability switches and catastrophic shifts. Theoretical Ecology 10 (2017), 235-244.

[16] J.M.R. Parrondo. How to cheat a bad mathematician. in EEC HC\&M Network on Complexity and Chaos (\#ERBCHRX-CT940546), ISI, Torino, Italy (1996). Unpublished. 
[17] J. Palis. Vector fields generate few diffeomorphisms. Bulletin of the American Mathematical Society 80 (1974), 503-505.

[18] M. Pireddu, F. Zanolin. Chaotic dynamics in the Volterra predator-prey model via linked twist maps. Opuscula Mathematica 28 (2008), 567-592.

[19] D. Xiao. Dynamics and bifurcations on a class of population model with seasonal constant-yield harvesting. Discrete and Continuous Dynamical Systems -series B 21 (2016), 699-719.

[20] C. Xu, M. S. Boyce, D. J. Daley. Harvesting in seasonal environments. Journal of Mathematical Biology 50 (2005), 663-682.

[21] X. Zhang. Embedding smooth diffeomorphisms in flows. Journal of Differential Equations 248 (2010), 1603-1616.

[22] X. Zhang. The embedding flows of $\mathcal{C}^{\infty}$ hyperbolic diffeomorphisms. Journal of Differential Equations 250 (2011), 2283-2298. 